\title{
Codoping: A possible pathway for inducing ferromagnetism in $\mathrm{ZnO}$
}

\author{
N. N. Lathiotakis* \\ Theoretical and Physical Chemistry Institute, NHRF, 48 Vassileos Constantinou, 11635 Athens, Greece \\ Antonis N. Andriotis ${ }^{\dagger}$ \\ Institute of Electronic Structure and Laser, FORTH, P.O. Box 1527, 71110 Heraklio, Crete, Greece

\begin{abstract}
Madhu Menon
Department of Physics and Astronomy, University of Kentucky, Lexington, Kentucky 40506-0055, USA and Center for Computational Sciences, University of Kentucky, Lexington, Kentucky 40506-0045, USA

(Received 8 April 2008; revised manuscript received 10 October 2008; published 24 November 2008)
\end{abstract}

\begin{abstract}
A detailed $a b$ initio (DFT/GGA and DFT/GGA $+U$ ) investigation of codoping $\mathrm{ZnO}$ with $\mathrm{Co}$ and $\mathrm{Cu}$ ions reveals the role of $\mathrm{Cu}$ and the existence of optimum structural dopant configurations which could lead to the enhancement of ferromagnetism. The role of the $\mathrm{Cu}$ ions in $\mathrm{Zn}(\mathrm{Co}, \mathrm{Cu}) \mathrm{O}$ is to act as superexchange mediators while causing a remote delocalization through the hybridization of the $\mathrm{Cu} d_{3 z^{2}-r^{2}}$ spin-majority states with the $\mathrm{O} p$ states, thereby enhancing the ferromagnetism. The ferromagnetic state is found to be more favorable than the antiferromagnetic one if the $\mathrm{Cu}-d$ band is spin splitted. Such a process is reminiscent of the conventional RKKY interaction among magnetic impurities embedded in a metal.
\end{abstract}

DOI: 10.1103/PhysRevB.78.193311

PACS number(s): 71.20.-b, 73.20.Hb, 75.30.Et, 75.30.Hx

The role of defects in developing ferromagnetism (FM) is well known. In particular, the study of doped $\mathrm{ZnO}$ has attracted much attention lately. Electron paramagnetic resonance (EPR) measurements has led to the conclusion that the observed magnetic signal from $n$-type samples of $\mathrm{Co}^{++}$-doped $\mathrm{ZnO}$ [to be denoted as $\mathrm{Zn}(\mathrm{Co}) \mathrm{O}$ ] should be attributed to states other than the $3 d^{7} \mathrm{Co}$ states, ${ }^{1}$ while magnetic circular dichroism (MCD) has shown that roomtemperature ferromagnetism in $\mathrm{Zn}(\mathrm{Co}) \mathrm{O}$ could be attributed to the hybridization of the conduction band of the doped $\mathrm{ZnO}$ with some of the $d$ electrons of the magnetic ions. ${ }^{2}$ Magnetism was also observed in $\mathrm{ZnO}$ doped with most of the other $3 d$ elements, among which the magnetism of the low-doped $\mathrm{Zn}(\mathrm{Mn}) \mathrm{O}$ leads to the conclusion that it can be induced by either localized defect states or band states. ${ }^{2}$

From the viewpoint of theory, the outcome for the relative stability of the ferromagnetic (FM) and antiferromagnetic (AFM) states of the Co-doped $\mathrm{ZnO}$ depends on how accurately the $e-e$ correlations are treated by the computational method used. ${ }^{3}$ Thus, some of these $n n$ Co configurations were found to exhibit FM and some others AFM ground state according to the density-functional theory (DFT) results within the local-density approximation (LDA). However, in the $\mathrm{LDA}+U$ approximation all the $n n$ Co configurations studied were found to prefer the AFM ordering., 3,4

On the other hand, the ferromagnetic exchange interaction found between $\mathrm{Cu}$ ions, in the $\mathrm{Cu}$-doped $\mathrm{ZnO}$, is attributed to the hole states delocalized via a $p$ - $d$ hybridization, ${ }^{5}$ leading to the conclusion that the existence of holes and metallicity are the necessary conditions to obtain high ferromagnetic stability. ${ }^{5}$ This conclusion is at odds with the findings of $\mathrm{Ye}$ et al. ${ }^{6}$ who demonstrated that the ferromagnetism in $\mathrm{Cu}-$ doped $\mathrm{ZnO}$ is not related to the hole density but rather to the inherent properties of the $\mathrm{CuO}_{4}$ tetrahedra and the associated hybridization among them.

In our recent reports, we pointed out the common origin of magnetism in the nontraditional inorganic materials [as, for example, that of some diluted magnetic semiconductors (DMSs), e.g., $\mathrm{Zn}(\mathrm{Co}) \mathrm{O}]$ and that of the $\mathrm{C}_{60}$-based polymers. $^{7-10}$ More specifically, the magnetism of these materials was attributed to the simultaneous presence of two kinds of defects (of structural/topological or of substitutional-impurity type) forming chains of alternating effective donor $(D)$ and acceptor $(A)$ crystal sites, ${ }^{11}$ which could induce selective remote delocalization, leading to significant charge- and spin-density transfers which take the form of charge-density-like waves (CDWs) and spin-densitylike waves (SDWs). ${ }^{12}$

Recently, codoping, i.e., the simultaneous presence of two kinds of defects, has attracted attention primarily because of the possibility of using it to tailor the position and occupancy of the Fermi energy $\left(E_{F}\right)$ of the doped DMS. ${ }^{13-19}$ Thus, codoping with shallow acceptors and donors was viewed as a potential means to specify the availability of carriers to mediate ferromagnetism. ${ }^{13-18}$

In the present work, we report results of a detailed investigation of the codoped $\mathrm{Zn}(\mathrm{Co}, \mathrm{Cu}) \mathrm{O}$ DMS and demonstrate the key role of the $\mathrm{Cu}^{+}$codopants in developing the magnetic features of the $\mathrm{Zn}(\mathrm{Co}, \mathrm{Cu}) \mathrm{O}$ system. We performed a DFT plane-wave calculation ${ }^{20}$ with ultrasoft pseudopotentials using the generalized gradient approximation (GGA) functional and the GGA $+U$ approximation. The supercell consisted of hexagonal $3 \times 3 \times 3$ cell containing 108 atoms. The supercell size is large enough to allow us to investigate a large number of different dopant and codopant geometric structures and isolate the interaction between impurities (dopant concentrations 4\%-5\%). Due to the big size of the supercell, we considered only two $k$ points, for which we chose the $k$ points introduced by Chadi. ${ }^{21}$ We verified the adequacy of this choice by increasing the number of $k$ points. No significant differences in the corresponding electron DOS's were found by going to a $4 \times 4 \times 4$ Monkhorst-Pack. Also, the differences in total energy between FM and AFM configurations (which is the quantity of interest) was found 
to change by less than $10 \%$ if the number of $k$ points were increased to eight. The kinetic-energy cutoff for the charge density and the potential was chosen to be 200 Ryd, and a Gaussian smearing parameter equal to $10^{-2}$ was used as a result of the small metallicity introduced by the doping. For all the structures, the spin-up and spin-down densities were fully converged.

In order to improve upon the deficiencies of the DFT/ GGA (i.e., underestimation of the band gap and the overestimation of the $\mathrm{Zn} d \mathrm{O} p$ hybridization ${ }^{22}$ ), we used the $\mathrm{LDA}+U$ approach. Our choice for the Hubbard $U$ parameters is $U_{\mathrm{Zn}}=6.5 \mathrm{eV}, U_{\mathrm{Co}}=2.5 \mathrm{eV}$, and $U_{\mathrm{Cu}}=1.0 \mathrm{eV}$. With this choice, the positions of the $\mathrm{Zn} d$ and the Co $d$ impurity bands are in best agreement with experiment. ${ }^{23}$ Our values for Hubbard $U$ correspond to the $U-J$ differences, where $U$ and $J$ denote the intrasite Coulomb and exchange interactions, respectively, and compare well with other theoretical investigations. $^{24-26}$ It should be noted, however, that the comparison among the various sets of the $U$ parameters used by various LSDA $+U$ implementations is meaningful only if it refers to similar implementations. More precisely, it depends strongly on the pseudopotential choice, the normalization of the atomic wave functions, the way occupancies and site-projected terms are evaluated, as well as the computational characteristics of each computer code that is employed. ${ }^{27}$ It should further be noted that the employment of the LSDA $+U$ level with $U$ terms for the $d$ states only does not improve upon the energy gap. This can be achieved by including $U$ terms for the $p$ electrons as well and, in particular, for the $\mathrm{O} p$ states. Recent investigations confirm that in this case the FM coupling prevails upon doping with carriers. $^{26}$

In the $3 \times 3 \times 3 \mathrm{ZnO}$ superlattice structure, we first substitute two of the $\mathrm{Zn}$ atoms with Co atoms forming four different structures: $\mathrm{C} 1, \mathrm{C} 2, \mathrm{D}$, and $\mathrm{E}$. In the first two $(\mathrm{C} 1$ and C2), the two Co impurities are first neighbors. In the first (C1), they lie on the same $\mathrm{Zn}$ plane perpendicular to the hexagonal $c$ axis, while in the second they lie on two adjacent planes. In the third and fourth (D and E), the Co atoms lie in second- and third-nearest-neighbor Zn planes, respectively. These structures were further doped with a $\mathrm{Cu}$ atom in various positions. All these structures with a codopant $\mathrm{Cu}$ atom are shown in Fig. 1. The numbering following the $\mathrm{Cu}$ atoms in the figure denotes the number of the $\mathrm{Zn}$ atom substituted by $\mathrm{Cu}$ in our supercell.

Our detailed analysis has shown that the Co $3 d$, the O $2 p$, and the $\mathrm{Cu} 3 d$ projected bands all span the same energy space, just below the Fermi energy for the majority spin and cross the Fermi energy for the minority spin (see Fig. 2). Thus, the interaction of the Co magnetic $d$ states involves their hybridization with the $\mathrm{O} 2 p$ and $\mathrm{Cu} 3 d$ states, while both $\mathrm{O}$ and $\mathrm{Cu}$ atoms acquire significant net magnetic moments (see below). In addition to these observations, some important additional trends can also be obtained from our results. In particular: (i) The FM enhancement is found in structural configurations in which the two Co atoms are located in different $\mathrm{Zn}$ planes in the $\mathrm{Zn}$ sublattice within the range of third $n n$. (ii) The $\mathrm{Cu}$-induced FM enhancement is more pronounced when the $\mathrm{Cu}$ atoms are located between the Co atoms (e.g., D-Cu55, D-Cu41) or in a highly symmet-

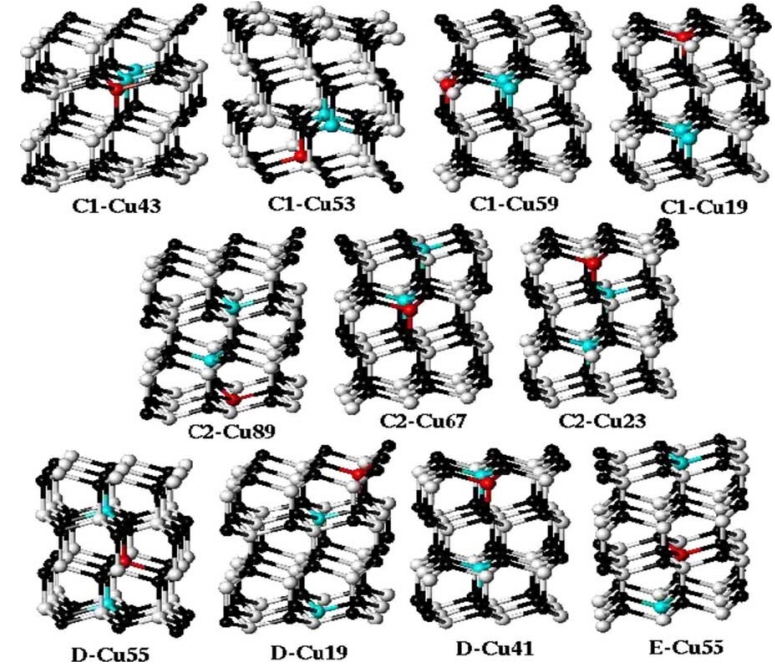

FIG. 1. (Color online) The structures of $\mathrm{ZnO}$ with the impurity $\mathrm{Co}$ atoms (blue/light gray) and the $\mathrm{Cu}$ impurities (red/dark gray).

ric position in the proximity of $\mathrm{Co}$ (e.g., $\mathrm{C} 1-\mathrm{Cu} 53$ ). (iii) Comparing the FM and the AFM states of a particular structure, it is observed that the structure for which the FM state is more stable corresponds to an AFM state in which the magnetic moment on the $\mathrm{Cu}$ atom is zero (e.g., D-Cu55). The FM and AFM states become degenerate if the splitting of the $\mathrm{Cu} d$ band is the same for both FM and AFM states (e.g., $\mathrm{D}-\mathrm{Cu} 19)$. Thus, the FM state becomes more favorable because the $\mathrm{Cu} d$ band is spin splitted. When the AFM state also supports such a splitting, then it is either favorable (like C1-Cu59) or the FM and AFM states are almost degenerate (like D-Cu19) (see Fig. 2). (iv) Magnetic enhancement
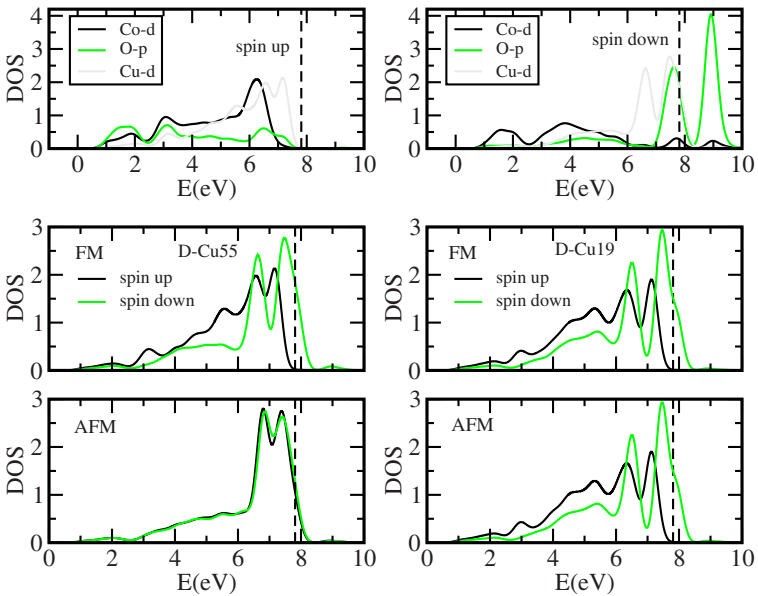

FIG. 2. (Color online) Top panel: The Co- $d-$, O-p-, and $\mathrm{Cu}-d$-projected DOS for the D-Cu55 structure (of Fig. 1). The Fermi energy is indicated by the vertical dashed line. The position of the bands in the energy scale supports a strong hybridization of these bands. The oxygen atom chosen for the projection is the one neighboring both the $\mathrm{Co}$ and $\mathrm{Cu}$ atoms. Bottom (double) panel: The $\mathrm{Cu}-d$-projected DOS for D-Cu55 (left) compared to D-Cu19 (right) for the FM and AFM states demonstrating the lack of splitting of the former in contradistinction to the splitting of the latter for the AFM states. The lack of splitting in the AFM configuration leads to strong FM behavior. 
TABLE I. Results for the structures shown in Fig. 1 obtained using the DFT/GGA for the total-energy difference between the AFM and FM configurations, the total and absolute magnetization (in Bohr magnetons), and the $\mathrm{Cu} d$-states projected magnetization for: (i) two substitutional Co impurities in $\mathrm{ZnO}$ and (ii) $\mathrm{ZnO}$ codoped with $\mathrm{Co}$ and $\mathrm{Cu}$. All magnetizations are in Bohr magnetons. Corresponding results derived using the DFT/GGA $+U$ level of approximation are shown in parentheses.

\begin{tabular}{|c|c|c|c|c|c|c|c|}
\hline \multirow[b]{2}{*}{ Structure } & \multirow{2}{*}{$\begin{array}{c}\Delta E(\mathrm{FM}-\mathrm{AFM}) \\
(\mathrm{meV})\end{array}$} & \multicolumn{2}{|c|}{ Magnetization } & \multicolumn{2}{|c|}{$\begin{array}{c}\text { Absolute } \\
\text { magnetization }\end{array}$} & \multicolumn{2}{|c|}{$\begin{array}{c}\mathrm{Cu} \\
\text { magnetization }\end{array}$} \\
\hline & & FM & AFM & FM & AFM & FM & AFM \\
\hline \multicolumn{8}{|c|}{ Only two Co impurities: } \\
\hline $\mathrm{C} 1$ & 42.50 & 6.0 & 0.0 & 6.11 & 5.87 & & \\
\hline (C1) & $(45.34)$ & $(6.0)$ & $(0.00)$ & $(6.10)$ & $(5.90)$ & & \\
\hline $\mathrm{C} 2$ & 0.716 & 6.0 & 0.0 & 6.12 & 5.95 & & \\
\hline$(\mathrm{C} 2)$ & $(14.64)$ & $(6.0)$ & $(0.00)$ & $(6.10)$ & $(5.90)$ & & \\
\hline $\mathrm{D}$ & -0.802 & 6.0 & 0.0 & 6.14 & 6.11 & & \\
\hline (D) & $(-0.400)$ & $(6.0)$ & $(0.00)$ & $(6.10)$ & $(6.10)$ & & \\
\hline $\mathrm{E}$ & -0.026 & 6.0 & 0.0 & 6.14 & 6.13 & & \\
\hline \multicolumn{8}{|c|}{ With $\mathrm{Cu}$ co-doping: } \\
\hline D-Cu55 & -65.9 & 7.08 & 0.05 & 7.25 & 6.90 & 0.47 & 0.03 \\
\hline (D-Cu55) & $(-44.2)$ & $(7.00)$ & $(0.05)$ & $(7.14)$ & $(6.76)$ & $(0.47)$ & $(0.03)$ \\
\hline D-Cu19 & -1.9 & 7.06 & 0.78 & 7.24 & 7.20 & 0.52 & 0.52 \\
\hline (D-Cu19) & $(87.5)$ & $(7.00$ & $1.00)$ & $(7.15)$ & $(7.13)$ & $(0.57)$ & $(0.65)$ \\
\hline D-Cu41 & -71.8 & 7.05 & 0.01 & 7.23 & 6.87 & 0.47 & 0.06 \\
\hline $\mathrm{C} 1-\mathrm{Cu} 43$ & -4.7 & 7.05 & 0.01 & 7.20 & 6.56 & 0.45 & 0.01 \\
\hline $\mathrm{C} 1-\mathrm{Cu} 53$ & -56.8 & 7.03 & 0.01 & 7.18 & 6.45 & 0.49 & 0.00 \\
\hline (C1-Cu53) & $(-125.3)$ & $(7.00)$ & $(0.00)$ & $(7.15)$ & $(6.37)$ & $(0.63)$ & $(0.00)$ \\
\hline C1-Cu59 & 33.9 & 7.03 & 0.74 & 7.18 & 6.91 & 0.50 & 0.49 \\
\hline (C1-Cu59) & $(7.4)$ & $(7.00)$ & $(1.00)$ & $(7.15)$ & $(6.91)$ & $(0.65)$ & $(0.62)$ \\
\hline $\mathrm{C} 1-\mathrm{Cu} 19$ & -22.8 & 7.03 & 0.02 & 7.18 & 6.62 & 0.50 & 0.04 \\
\hline C2-Cu89 & -2.1 & 7.05 & 0.78 & 7.22 & 7.0 & 0.51 & 0.51 \\
\hline (C2-Cu89) & (14.9) & $(7.00)$ & $(1.00)$ & $(7.15)$ & $(6.98)$ & $(0.65)$ & $(0.64)$ \\
\hline C2-Cu67 & -35.2 & 7.04 & 0.71 & 7.20 & 6.87 & 0.46 & 0.45 \\
\hline (C2-Cu67) & $(8.3)$ & $(7.00)$ & $(1.00)$ & $(7.14)$ & $(6.89)$ & $(0.62)$ & $(0.64)$ \\
\hline $\mathrm{C} 2-\mathrm{Cu} 23$ & -99.2 & 7.02 & 0.03 & 7.19 & 6.29 & 0.49 & 0.00 \\
\hline E-Cu55 & -0.8 & 7.07 & 0.75 & 7.25 & 7.23 & 0.51 & 0.51 \\
\hline
\end{tabular}

seems to have its origin in the inter-Zn-plane interactions. The intra-Zn-plane interactions seem to be of minor effect in the development of ferromagnetism or lead to AFM states (e.g., C1-Cu43, C1-Cu59). (v) The magnetic configurations which show enhanced magnetic features are by far the most stable ones.

Our findings are summarized in Table I. In the absence of any additional impurities, and within the DFT/GGA, the AFM state seems to be more stable for the two Co atoms if they are first neighbors in the $\mathrm{Zn}$ sublattice. This behavior is even more pronounced by the introduction of Hubbard $U$ correction (see "Only two Co impurities," top part of Table I). This result is in agreement with other calculations. ${ }^{3,28,29}$ For larger interatomic distances, the FM configuration is favorable but the energy difference between the two states is very small. In the presence of an additional $\mathrm{Cu}$ impurity we observe (within the DFT/GGA) a significant enhancement of the stability of the FM state versus the AFM one. The FM coupling was found more stable in all the geometries studied except one (see bottom part of Table I). On the other hand, while the stability of the FM phase is reduced relative to that of the AFM one at the LSDA $+U$ level, it does not affect our conclusions indicating that the participation of the $\mathrm{Cu}$ codopant in the hybridization of the Co $3 d-\mathrm{O} 2 p$ orbitals dominates the $e$ - $e$ correlations. Again, in these cases the FM arrangement is observed when $\mathrm{Cu}$ is not magnetized in the AFM configuration. A noticeable difference between the DFT/GGA and the DFT/GGA $+U$ results is that the acquired magnetic moment on $\mathrm{Cu}$ is found larger in the DFT/GGA $+U$ case. That is, when AFM prevails, the total magnetization is higher than for pure GGA by 0.3 Bohr magnetons.

Our results reconfirm previous findings ${ }^{12,29}$ that codoping $\mathrm{Zn}(\mathrm{Co}) \mathrm{O}$ with $\mathrm{Cu}$ results in stable FM ground-state configurations. However, the picture we obtained for the role of $\mathrm{Cu}^{+}$ 
in enhancing the FM stability between the magnetic impurities is quite different. In particular, it is found that the role of $\mathrm{Cu}^{+}$is analogous to that played by the free electron in the development of the Ruderman-Kittel-Kasuya-Yosida (RKKY) interaction. The $\mathrm{Cu}^{+}$ion, being spin polarized by the presence of the magnetic impurities (as shown in Fig. 2), "communicates" the disturbance to its neighboring magnetic impurities, thus, mediating an indirect magnetic interaction among them. Similar interpretation can also be given for the results of Ye et al., ${ }^{6}$ as seen in their Table I, where a change in polarization of the interstitial charge can be observed when switching from the FM to the AFM state. Given the half-metallic character of the $\mathrm{Zn}(\mathrm{Co}, \mathrm{Cu}) \mathrm{O}$ system (see Fig. 2, top panel), a small contribution of the conventional RKKY mechanism mediating ferromagnetism can also be expected; however, not to the extent of the spin-polarization mechanism of the mediating $\mathrm{Cu}$ ions due mainly to the large separations between the Co ions. It could then be said that the $\mathrm{Zn}(\mathrm{Co}, \mathrm{Cu}) \mathrm{O}$ system gives us a clear demonstration of the way an RKKY-like mechanism for ferromagnetism evolves in a semiconducting system, i.e., in the absence of charge carriers, taking the form of a superexchange interaction.

In contradistinction to the free-electron case, the $\mathrm{Cu}$ mediated interaction is highly anisotropic as evidenced by the strength of the calculated FM coupling for the various configurations shown in Table I. We attribute this behavior to the band filling of the $d$ orbitals of $\mathrm{Cu}$ and their directionality. This is supported by our findings that the $\mathrm{Cu} d_{3 z^{2}-r^{2}}$ states (the $z$ axis taken to be along the hexagonal close pack $c$ axis) split more than the remaining $d$ states of $\mathrm{Cu}$. In the cases that ferromagnetism is pronounced, no splitting of these states is found in the AFM state. In the AFM state, the $\mathrm{Cu} d_{3 z^{2}-r^{2}}$ are almost degenerate with the other $d$ states, especially the $d_{x^{2}-y^{2}}$ and the $d_{x y}$. In the FM state, on the other hand, this degeneracy is lifted, leading to a lowering of energy.

The superexchange form of the interaction of the Co at- oms follows a path through the $\mathrm{O}$ and $\mathrm{Cu}$ atoms. The key mechanism is the hybridization of the Co $d$ states with the $\mathrm{O} p$ and subsequently with the $\mathrm{Cu} d$ states. In the structures with pronounced ferromagnetism, the hybridization of the $\mathrm{Cu} d$ states with the $\mathrm{O} p$ is particularly enhanced. That effect is assisted by the downwards shift of the $\mathrm{Cu} d_{3 z^{2}-r^{2}}$ spinmajority band which overlaps (in energy scale) with the $\mathrm{O} p$ band. This mechanism was more evident for the structures D-Cu55, C1-Cu53, and C2-Cu23, which are all found to be strongly FM. Clearly, the enhancement of the hybridization results in additional delocalization of the $\mathrm{Cu} d_{3 z^{2}-r^{2}}$ bands analogous to that found in the case of $\mathrm{C}_{60}$-based magnetic polymers. ${ }^{12}$ I.e., the $p$ - $d$ hybridization induces a remote electron delocalization which mediates the Co-Co interaction. This observation is in partial support of the conclusion arrived at by Ye et al., ${ }^{6}$ who attributed the magnetic features of the $\mathrm{Zn}(\mathrm{Cu}) \mathrm{O}$ to the inherent properties of the $\mathrm{CuO}_{4}$ tetrahedra and their coupling.

In conclusion, it has been demonstrated that in codoped $\mathrm{Zn}(\mathrm{Co}, \mathrm{Cu}) \mathrm{O}$ there exist optimum structural dopant configurations which lead to the enhancement of ferromagnetism. The role of the $\mathrm{Cu}^{+}$ions in $\mathrm{Zn}(\mathrm{Co}, \mathrm{Cu}) \mathrm{O}$ is to act as superexchange mediators by causing a remote delocalization through the hybridization of the $\mathrm{Cu} d_{3 z^{2}-r^{2}}$ spin-majority states with the $\mathrm{O} p$ states, thereby enhancing the ferromagnetism. Based on our findings we propose that $\mathrm{ZnO}$ grown by beam epitaxy, during which the doping of $\mathrm{Co}$ and $\mathrm{Cu}$ ions takes place in successive layers along the $\mathrm{Zn}$ planes of the $\mathrm{Zn}$ sublattice, could lead to a material with enhanced magnetic properties.

The present work is supported by U.S.-ARO (Grant No. W911NF-05-1-0372), DOE (Grants No. DE-FG0200ER45817 and No. DE-FG02-07ER46375), Deutsche Forschungsgemeinschaft (Program No. SPP 1145), and by the NANOQUANTA Network of Excellence.

\footnotetext{
*lathiot@physik.fu-berlin.de

†andriot@iesl.forth.gr

*madhu@ccs.uky.edu

${ }^{1}$ P. Sati et al., Phys. Rev. Lett. 96, 017203 (2006).

${ }^{2}$ J. R. Neal et al., Phys. Rev. Lett. 96, 197208 (2006).

${ }^{3}$ S. J. Hu et al., Phys. Rev. B 73, 245205 (2006).

${ }^{4}$ T. Chanier et al., Phys. Rev. B 73, 134418 (2006).

${ }^{5}$ L. M. Huang et al., Phys. Rev. B 74, 075206 (2006).

${ }^{6}$ L. H. Ye et al., Phys. Rev. B 73, 033203 (2006).

${ }^{7}$ A. N. Andriotis et al., Phys. Rev. Lett. 90, 026801 (2003).

${ }^{8}$ A. N. Andriotis et al., Clusters and Nano-Assemblies: Physical and Biological Systems, edited by P. Jena and S. N. Khanna and B. K. Rao (World Scientific, Singapore, 2005).

${ }^{9}$ A. N. Andriotis et al., J. Phys.: Condens. Matter 17, L35 (2005).

${ }^{10}$ A. N. Andriotis et al., Europhys. Lett. 72, 658 (2005).

${ }^{11}$ H. M. McConnell, Proc. Robert A. Welch Found. Conf. Chem. Res. 11, 144 (1967).

${ }^{12}$ A. N. Andriotis et al., Int. J. Nanotechnol. 6, 164 (2009).

${ }^{13}$ M. J. Reed et al., Appl. Phys. Lett. 86, 102504 (2005).

${ }^{14}$ K. R. Kittilstved et al., Phys. Rev. Lett. 94, 147209 (2005).
}

${ }^{15}$ N. Ozaki et al., Appl. Phys. Lett. 87, 192116 (2005).

${ }^{16}$ M. H. Kane et al., J. Cryst. Growth 287, 591 (2006).

${ }^{17}$ N. Ozaki et al., Phys. Rev. Lett. 97, 037201 (2006).

${ }^{18}$ S. Kuroda et al., Nature Mater. 6, 440 (2007).

${ }^{19}$ T. Dietl, Nature Mater. 5, 673 (2006).

${ }^{20}$ Plane-Wave Self-Consistent-Field (PWSCF) code (http:// www.pwscf.org/).

${ }^{21}$ D. J. Chadi et al., Phys. Rev. B 8, 5747 (1973).

${ }^{22}$ S. Massidda et al., Phys. Rev. B 52, R16977 (1995).

${ }^{23}$ B. Sanyal et al., J. Appl. Phys. 103, 07D130 (2008).

${ }^{24}$ S. Z. Karazhanov et al., Phys. Rev. B 75, 155104 (2007).

${ }^{25}$ S. Lany et al., Phys. Rev. B 77, 241201(R) (2008).

${ }^{26}$ A. Walsh et al., Phys. Rev. Lett. 100, 256401 (2008).

${ }^{27} \mathrm{Hu}$ et al. (Ref. 3) used implementation identical to the present work and obtained values for $U$ that are substantially smaller. This discrepancy can be traced back to the normalization of the pseudopotential atomic wave functions.

${ }^{28}$ E. C. Lee et al., Phys. Rev. B 69, 085205 (2004).

${ }^{29}$ R. Janisch et al., J. Phys.: Condens. Matter 17, R657 (2005). 